\title{
An Improved Exponential Type Estimator Of Population Mean of Sensitive Variable Using Optional Randomized Response Technique
}

\author{
Lovleen Kumar Grover \\ Department of Mathematics, \\ Guru Nanak Dev University, Amritsar, Punjab, India \\ Email:lovleen_2@yahoo.co.in \\ Amanpreet Kaur \\ Department of Mathematics, \\ Guru Nanak Dev University, Amritsar, Punjab, India
}

\begin{abstract}
In this paper, we improve the efficiency of Koyuncu et al (2014)'s estimator of population mean of sensitive variable by replacing Traditional Randomized response technique with Optional Randomized response technique as suggested by Gupta et al (2014). The mean square error of proposed estimator is obtained, up to first order of approximation, and is compared with mean square error of various existing estimators theoretically as well as numerically.
\end{abstract}

Keywords: Auxiliary variable; bias; efficiency; mean square error; randomized response technique; simple random sampling without replacement; sensitive study variable; percent relative efficiency.

AMS Classification: 62D05

\section{Introduction}

We know that auxiliary information plays an important role to improve the efficiency of an estimator of parameter of interest when the study variable is sensitive or non-sensitive in nature. Bahl and Tuteja (1991), Grover and Kaur (2011), Singh and Solanki (2012) and many more authors used auxiliary information when the study variable is non sensitive whereas Sousa et al (2010), Gupta et al (2012), Koyuncu et al (2014), Kalucha et al (2015) and many more authors used auxiliary information in Randomized response technique (RRT) under traditional additive model when the study variable is sensitive. But auxiliary variable is non sensitive in both the situations. Some authors including Gupta et al (2010), Huang (2010), Gupta et al (2013), and Gupta et al (2014) have studied Optional RRT with modified additive model. Tarray and Singh (2017) have suggested optional RRT with new additive model. The importance of Optional RRT model lies in the fact that a question may not be sensitive for the entire population. One person consider a particular question as sensitive question and other may consider it non sensitive question. In an Optional RRT Model, scrambled answer is given by the respondent only if he/she consider the question is sensitive otherwise true answer is given by the respondent. Gupta et al (2014) suggested an efficient estimator of population mean of sensitive variable by replacing traditional RRT model used in Sousa et al (2010) and Gupta et al (2012) with Optional RRT model. In this article, we use Optional RRT model to improve the efficiency of an exponential type estimator suggested by Koyuncu et al (2014). Our proposed estimator is also more efficient than the estimators suggested by Gupta et al (2014). In this article, we will deal with the quantitative study variable, 
whereas some authors in the literature like Singh and Tarray (2014), Tarray et al (2015), etc studied optional randomized response model for qualitative study variable. To support theoretical results obtained, a numerical illustration is considered finally.

\section{Notations and existing estimators}

Consider a population $U=\left(U_{1}, U_{2}, \ldots, U_{N}\right)$ of size $N$ from which a sample of size $n$ is drawn using simple random sampling without replacement. Let $Y$ be the study variable which is sensitive in nature. Let $X$ be a non-sensitive auxiliary variable which is positively correlated with the study variable $Y$. Let $W$ be the sensitivity level of the asked sensitive question. The respondent gives the correct response for the auxiliary variable $X$ but has optional randomized response for variable $Y$. In this Optional RRT model, respondent gives the response as $Z=Y+S T$ for the study variable $Y$, where $T$ is a Bernoulli random variable with parameter $W$, so that $0 \leq W \leq 1$ and $S$ is a scrambling variable whose mean is assumed to be zero i.e. $E(S)=\bar{S}=0$ and its variance $\sigma_{S}^{2}$ is assumed to be known quantity. It is assumed that the variables $S$ and $T$ are two mutually independent variables which are further independent of variables $Y$ and $X$.

\section{Remark 2.1:}

If we take $W=1$ in the above model then it reduces to the traditional additive RRT model, and scrambled response is then written as $Z=Y+S$.

Now the population mean of variable $Z$ is given by $\bar{Z}=E(Z)=E(Y+S T)=E(Y)=$ $\bar{Y}=\mu_{Y Z}$ (say) as $E(S)=0$, where $\bar{Y}$ is the population mean of variable $Y$. The population variance of variable $Z$ is given by $S_{z}^{2}=V(Y+S T)=S_{y}^{2}+W S_{S}^{2}$. Let $C_{z}$ be the coefficient of variation of variable $Z$. So $C_{z}^{2}=C_{y}^{2}+W \frac{S_{s}^{2}}{\bar{Y}^{2}}$, where $C_{y}$ is the coefficient of variation of variable $Y$. Let $\rho_{z x}$ be the coefficient of correlation between variables $Z$ and $X$ so $\rho_{z x}=\frac{\rho_{y x}}{\sqrt{1+W \frac{s_{s}^{2}}{s_{y}^{2}}}}$, where $\rho_{y x}$ is the coefficient of correlation between variables $Y$ and $X, S_{y}^{2}=\frac{1}{N-1} \sum_{i=1}^{N}\left(y_{i}-\bar{Y}\right)^{2}$, and $S_{S}^{2}=\frac{1}{N-1} \sum_{i=1}^{N}\left(s_{i}-\bar{S}\right)^{2}$.

Taking also $S_{X}^{2}=\frac{1}{N-1} \sum_{i=1}^{N}\left(x_{i}-\bar{X}\right)^{2}$, where $\bar{X}$ is the population mean of auxiliary variable $X$. Let $C_{x}$ be the coefficient of variation of variable $X$.

\section{Remark 2.2:}

The estimate of sensitivity level $W$ in the above Optional RRT model may be obtained by using the same approach of Gupta et al (2014). According to them, the estimated value of $W$ is $\widehat{W}=\frac{\frac{1}{n} \sum_{i=1}^{n} z_{i}^{2}-\left\{\widehat{V}(y)+\left(\frac{1}{n} \sum_{i=1}^{n} z_{i}\right)^{2}\right\}}{E\left(s^{2}\right)}$, where $\widehat{V}(y)$ is the estimate of variance of y. They further found that

$\widehat{W}=\frac{\frac{1}{n} \sum_{i=1}^{n} z_{i}^{2}-\left\{\frac{1}{n} \sum_{i=1}^{n} z_{i}+\left(\frac{1}{n} \sum_{i=1}^{n} z_{i}\right)^{2}\right\}}{E\left(s^{2}\right)}$, when $Y$ is assumed to follow Poisson distribution and $\widehat{W}=\frac{\hat{S}_{z}^{2}-\left(C_{x} \frac{1}{n} \sum_{i=1}^{n} z_{i}\right)^{2}}{E\left(s^{2}\right)}$, when it is assumed that $C_{x}=C_{y}$. 
Assume that $\bar{X}$ is known. Now we consider estimator suggested by Koyuncu et al (2014) and various other estimators under Traditional RRT Model: $Z=Y+S$ and also various estimators suggested by Gupta et al (2014) under Optional RRT Model: $Z=Y+S T$. These estimators and their mean square errors, up to first order of approximation, are given in the following table.

\section{Table2.1: Existing estimators with their mean square errors under various RRT} models

\begin{tabular}{|c|c|c|c|c|}
\hline & \multicolumn{2}{|c|}{ Traditional RRT Model: $Z=Y+S$} & \multicolumn{2}{|c|}{ Optional RRT Model: $Z=Y+S T$} \\
\hline & Estimator & $\begin{array}{lr}\text { Mean } & \text { Square } \\
\text { Error/Optimum } & \text { mean } \\
\text { square error } & \\
\end{array}$ & Estimator & $\begin{array}{l}\text { Mean Square } \\
\text { Error }\end{array}$ \\
\hline $\begin{array}{l}\text { Ordinary } \\
\text { unbiased } \\
\text { estimator }\end{array}$ & $\begin{array}{l}\text { Suggested by Sousa et } \\
\text { al (2010) } \\
\qquad \hat{\mu}_{Y Z}=\frac{1}{n} \sum_{i=1}^{n} z_{i}=\bar{z}\end{array}$ & $\lambda\left(S_{y}^{2}+S_{s}^{2}\right)$ & $\begin{array}{l}\text { Suggested by } \\
\text { Gupta et al } \\
(2014) \\
\hat{\mu}_{Y Z} \\
=\frac{1}{n} \sum_{i=1}^{n} z_{i}=\bar{z}\end{array}$ & $\lambda\left(S_{y}^{2}+W S_{s}^{2}\right)$ \\
\hline $\begin{array}{l}\text { Ratio type } \\
\text { estimator }\end{array}$ & $\begin{array}{l}\text { Suggested by Sousa et } \\
\text { al (2010) } \\
\qquad \hat{\mu}_{R Z}=\bar{z} \frac{\bar{X}}{\bar{x}}\end{array}$ & $\begin{array}{l}\lambda \bar{Y}^{2}\left(C_{y}^{2}+\frac{S_{s}^{2}}{\bar{Y}^{2}}+C_{x}^{2}\right. \\
\left.-2 \rho_{y x} C_{x} C_{y}\right)\end{array}$ & $\begin{array}{l}\text { Suggested by } \\
\text { Gupta et al } \\
\begin{array}{l}(2014) \\
\hat{\mu}_{R Z}=\bar{z} \frac{\bar{X}}{\bar{x}}\end{array}\end{array}$ & $\begin{array}{l}\lambda \bar{Y}^{2}\left(C_{y}^{2}\right. \\
+W \frac{S_{s}^{2}}{\bar{Y}^{2}}+C_{x}^{2} \\
\left.-2 \rho_{y x} C_{x} C_{y}\right)\end{array}$ \\
\hline $\begin{array}{l}\text { Regression } \\
\text { type } \\
\text { estimator }\end{array}$ & $\begin{array}{l}\text { Suggested by Gupta et } \\
\text { al (2012) } \\
\begin{array}{r}\hat{\mu}_{\text {Regz }}=\bar{z}+\hat{\beta}_{z x}(\bar{X} \\
\quad-\bar{x})\end{array}\end{array}$ & $\lambda S_{y}^{2}\left\{\left(1+\frac{S_{s}^{2}}{S_{y}^{2}}\right)-\rho_{y x}^{2}\right\}$ & $\begin{array}{l}\text { Suggested by } \\
\text { Gupta et al } \\
(2014) \\
\quad \hat{\mu}_{R e g z} \\
\quad=\bar{z} \\
\quad+\hat{\beta}_{z x}(\bar{X}-\bar{x})\end{array}$ & $\begin{array}{l}\lambda S_{y}^{2}\{(1 \\
\left.+W \frac{S_{s}^{2}}{S_{y}^{2}}\right) \\
\left.-\rho_{y x}^{2}\right\}\end{array}$ \\
\hline $\begin{array}{l}\text { Generalized } \\
\text { regression- } \\
\text { cum-ratio } \\
\text { estimator }\end{array}$ & $\begin{array}{l}\text { Suggested by Gupta et } \\
\text { al (2012) } \\
\quad \hat{\mu}_{G R R Z} \\
\quad=\left\{k_{1} \bar{z}\right. \\
\left.\quad+k_{2}(\bar{X}-\bar{x})\right\}\left(\frac{\bar{X}}{\bar{x}}\right)\end{array}$ & $\frac{\operatorname{MSE}\left(\hat{\mu}_{\operatorname{Reg} Z}\right)\left(1-\lambda C_{x}^{2}\right)}{\frac{M S E\left(\widehat{\mu}_{\operatorname{Reg} Z}\right)}{\bar{Y}^{2}}+\left(1-\lambda C_{x}^{2}\right)}$ & --- & --- \\
\hline $\begin{array}{l}\text { Exponential } \\
\text { type } \\
\text { estimator }\end{array}$ & $\begin{array}{l}\text { Suggested by Koyuncu } \\
\text { et al }(2014) \\
\quad \hat{\mu}_{\exp z} \\
\quad=\left[w_{1} \bar{z}\right. \\
\quad+w_{2}(\bar{X} \\
\quad-\bar{x})] \exp \left(\frac{\bar{X}-\bar{x}}{\bar{X}+\bar{x}}\right)\end{array}$ & $\begin{aligned} M S E\left(\hat{\mu}_{R e g Z}\right)- & T_{1 Z} \\
& -T_{2 Z}\end{aligned}$ & --- & --- \\
\hline
\end{tabular}

where $\lambda=\frac{1}{n}-\frac{1}{N}, \hat{\beta}_{z x}$ and $\hat{\beta}_{z x}$ are estimates of regression coefficients,

$T_{1 Z}=\frac{\frac{\left\{M S E\left(\widehat{\mu}_{R e g Z}\right)\right\}^{2}}{\bar{Y}^{2}}}{1+\frac{M S E\left(\widehat{\mu}_{\text {RegZ }}\right)}{\bar{Y}^{2}}}>0$ and $\quad T_{2 Z}=\frac{\lambda C_{x}^{2}\left\{M S E\left(\widehat{\mu}_{R e g Z}\right)+\lambda \frac{1}{16} C_{x}^{2} \bar{Y}^{2}\right\}}{4\left\{1+\frac{M S E\left(\widehat{\mu}_{R e g Z}\right)}{\bar{Y}^{2}}\right\}}>0$ 


\section{Proposed exponential estimator and its properties}

If $\bar{X}$ is known then we propose the following estimator of $\bar{Y}$ by replacing scrambled variable $Z=Y+S$ in Koyuncu et al (2014) with the scrambled variable $Z=Y+S T$ :

$$
\hat{\mu}_{\text {expZ }}=\left[m_{1} \bar{z}+m_{2}(\bar{X}-\bar{x})\right] \exp \left(\frac{\bar{X}-\bar{x}}{\bar{X}+\bar{x}}\right)
$$

where $m_{1}$ and $m_{2}$ are suitable chosen constants.

The Bias and Mean square error, up to first order of approximation, are respectively given by

$$
\begin{gathered}
\operatorname{Bias}\left(\hat{\mu}_{\text {expz }}\right) \cong \bar{Y}\left\{\left(m_{1}-1\right)+\frac{\lambda m_{1}}{2}\left(\frac{3}{4} C_{x}^{2}-C_{z x}\right)\right\}+\frac{\lambda m_{2}}{2} \bar{X} C_{x}^{2} \\
\operatorname{MSE}\left(\hat{\mu}_{\text {expz }}\right) \cong \bar{Y}^{2}+m_{1} \bar{Y}^{2}\left\{\lambda\left(C_{z x}-\frac{3}{4} C_{x}^{2}\right)-2\right\}-m_{2} \bar{Y} \bar{X} \lambda C_{x}^{2}+2 m_{1} m_{2} \bar{Y} \bar{X} \lambda\left(C_{x}^{2}-\right. \\
\left.C_{z x}\right)+m_{1}^{2} \bar{Y}^{2}\left\{1+\lambda\left(C_{z}^{2}+C_{x}^{2}-2 C_{z x}\right)\right\}+m_{2}^{2} \bar{X}^{2} \lambda C_{x}^{2}
\end{gathered}
$$

When we minimise $\operatorname{MSE}\left(\hat{\mu}_{\text {expz }}\right)$ w.r.t. $m_{1}$ and $m_{2}$, then optimum values of $m_{1}$ and $m_{2}$ are obtained as follows

$$
\begin{aligned}
& m_{1}^{(o p t)}=\frac{1-\frac{1}{8} \lambda C_{x}^{2}}{1+\lambda C_{z}^{2}\left(1-\rho_{z x}^{2}\right)} \\
& m_{2}^{(o p t)}=\frac{\bar{Y}}{2 \bar{X}} \frac{-C_{x}^{2}+2 C_{z x}+\lambda C_{x}^{2}\left\{C_{z}^{2}\left(1-\rho_{z x}^{2}\right)+\frac{1}{4}\left(C_{x}^{2}-C_{z x}\right)\right\}}{C_{x}^{2}\left\{1+\lambda C_{z}^{2}\left(1-\rho_{z x}^{2}\right)\right\}}
\end{aligned}
$$

The minimum mean square error of $\hat{\mu}_{\text {expz }}$ corresponding to these optimum values of $m_{1}$ and $m_{2}$ is given by

$$
\begin{aligned}
& \operatorname{Min} \cdot \operatorname{MSE}\left(\hat{\mu}_{\text {expz }}\right)=\frac{\lambda \bar{Y}^{2} C_{z}^{2}\left(1-\rho_{z x}^{2}\right)}{1+\lambda C_{z}^{2}\left(1-\rho_{z x}^{2}\right)}-\frac{\lambda^{2} \bar{Y}^{2} C_{x}^{2}\left\{4 C_{z}^{2}\left(1-\rho_{z x}^{2}\right)+\frac{C_{x}^{2}}{4}\right\}}{16\left\{1+\lambda C_{z}^{2}\left(1-\rho_{z x}^{2}\right)\right\}} \\
& =\frac{\lambda S_{y}^{2}\left\{\left(1+W \frac{s_{s}^{2}}{s_{y}^{2}}\right)-\rho_{y x}^{2}\right\}}{1+\frac{\lambda S_{y}^{2}\left\{\left(1+W \frac{s_{s}^{2}}{s_{y}^{2}}\right)-\rho_{y x}^{2}\right\}}{\bar{Y}^{2}}}-\frac{\lambda^{2} C_{x}^{2}\left\{4 S_{y}^{2}\left\{\left(1+W \frac{S_{s}^{2}}{S_{y}^{2}}\right)-\rho_{y x}^{2}\right\}+\frac{\bar{Y}^{2} C_{x}^{2}}{4}\right\}}{16\left\{1+\frac{\lambda S_{y}^{2}\left\{\left(1+W S_{\frac{s}{2}}^{2}\right)-\rho_{y x}^{2}\right\}}{\bar{Y}^{2}}\right\}} \\
& =\frac{M S E\left(\hat{\mu}_{R e g Z}\right)}{1+\frac{M S E\left(\hat{\mu}_{\text {RegZ }}\right)}{\bar{Y}^{2}}}-\frac{\lambda C_{x}^{2}\left\{M S E\left(\hat{\mu}_{R e g Z}\right)+\lambda \frac{1}{16} C_{x}^{2} \bar{Y}^{2}\right\}}{4\left\{1+\frac{M S E\left(\hat{\mu}_{R e g Z}\right)}{\bar{Y}^{2}}\right\}} \\
& =\operatorname{MSE}\left(\hat{\mu}_{\text {Regz }}\right)-\frac{\frac{\left\{\operatorname{MSE}\left(\hat{\mu}_{\text {Regz }}\right)\right\}^{2}}{\bar{Y}^{2}}}{1+\frac{M S E\left(\hat{\mu}_{\text {Regz }}\right)}{\bar{Y}^{2}}}-\frac{\lambda C_{x}^{2}\left\{\operatorname{MSE}\left(\hat{\mu}_{\text {Regz }}\right)+\lambda \frac{1}{16} C_{x}^{2} \bar{Y}^{2}\right\}}{4\left\{1+\frac{M S E\left(\hat{\mu}_{\text {Regz }}\right)}{\bar{Y}^{2}}\right\}} \\
& =\operatorname{MSE}\left(\hat{\mu}_{R e g z}\right)-T_{1 Z}-T_{2 Z} \\
& \text { where } T_{1 Z}=\frac{\frac{\left\{M S E\left(\hat{\mu}_{\text {Regz }}\right)\right\}^{2}}{\bar{Y}^{2}}}{1+\frac{M S E\left(\hat{\mu}_{\text {Regz }}\right)}{\bar{Y}^{2}}}>0 \text { and } \quad T_{2 Z}=\frac{\lambda C_{x}^{2}\left\{M S E\left(\widehat{\mu}_{\text {Regz }}\right)+\lambda \frac{1}{16} C_{x}^{2} \bar{Y}^{2}\right\}}{4\left\{1+\frac{M S E\left(\widehat{\mu}_{\text {Regz }}\right)}{\bar{Y}^{2}}\right\}}>0 \text {. }
\end{aligned}
$$

\section{Remark 3.1:}

Under Optional RRT model with the scrambled variable $Z=Y+S T$, one can also proposed the following generalized regression-cum-ratio estimator: 
$\hat{\mu}_{G R R Z}=\left[d_{1} \bar{z}+d_{2}(\bar{X}-\bar{x})\right]\left(\frac{\bar{X}}{\bar{x}}\right)$, where $d_{1}$ and $d_{2}$ are suitable chosen constants.

The minimum mean square error of $\hat{\mu}_{G R R Z}$, upto first order of approximation, is given by

$$
\begin{gathered}
\operatorname{Min} . \operatorname{MSE}\left(\hat{\mu}_{G R R z}\right) \cong \frac{\bar{Y}^{2} C_{z}^{2}\left(1-\rho_{z x}^{2}\right) \lambda\left(1-\lambda C_{x}^{2}\right)}{C_{z}^{2}\left(1-\rho_{z x}^{2}\right) \lambda+\left(1-\lambda C_{x}^{2}\right)} \\
=\frac{S_{y}^{2}\left\{\left(1+W \frac{S_{s}^{2}}{S_{y}^{2}}\right)-\rho_{y x}^{2}\right\} \lambda\left(1-\lambda C_{x}^{2}\right)}{\frac{S_{y}^{2}\left\{\left(1+W \frac{S_{s}^{2}}{S_{y}^{2}}\right)-\rho_{y x}^{2}\right)}{\bar{Y}^{2}}+\left(1-\lambda C_{x}^{2}\right)} \\
=\frac{M S E\left(\widehat{\mu}_{\text {Regz }}\right)\left(1-\lambda C_{x}^{2}\right)}{\frac{M S E\left(\hat{\mu}_{\text {Regz }}\right)}{\bar{Y}^{2}}+\left(1-\lambda C_{x}^{2}\right)} .
\end{gathered}
$$

Here we have obtained the minimum mean square errors of the proposed estimators $\hat{\mu}_{\text {expz }}$ and $\hat{\mu}_{G R R z}$ in terms of $M S E\left(\hat{\mu}_{R e g z}\right)$ because this makes possible to perform easily the comparative study of mean square error of proposed estimator with that of the existing estimators.

\section{Comparison of the proposed estimator with the existing estimators}

Now we will compare the mean square error of proposed estimator with that of existing estimators under traditional additive RRT model and Optional RRT model. Now we have the following results:

(I) $\operatorname{MSE}\left(\hat{\mu}_{y z}\right)-\operatorname{Min} \cdot \operatorname{MSE}\left(\hat{\mu}_{\text {expz }}\right)=\lambda \bar{Y}^{2} C_{y}^{2} \rho_{y x}^{2}+T_{1 Z}+T_{2 Z}+T>0$, always

where $T=\frac{\bar{Y}^{2}\left(\frac{\lambda C_{x}^{2}}{4}-2\right)^{2}\left\{(1-W) \lambda C_{y}^{2} \frac{S_{s}^{2}}{S_{y}^{2}}\right\}}{4\left\{1+\frac{M S E\left(\widehat{\mu}_{R e g z}\right)}{\bar{Y}^{2}}\right\}\left\{1+\frac{M S E\left(\widehat{\mu}_{\text {Regz }}\right)}{\bar{Y}^{2}}\right\}} \geq 0$.

(II) $\operatorname{MSE}\left(\hat{\mu}_{R Z}\right)-\operatorname{Min} . \operatorname{MSE}\left(\hat{\mu}_{\text {expz }}\right)=\lambda \bar{Y}^{2}\left(\rho_{y x} C_{y}-C_{x}\right)^{2}+T_{1 Z}+T_{2 Z}+T>0$, always.

(III) $\operatorname{MSE}\left(\hat{\mu}_{\text {Regz }}\right)-\operatorname{Min} \cdot \operatorname{MSE}\left(\hat{\mu}_{\exp z}\right)=T_{1 Z}+T_{2 Z}+T>0$, always.

(IV) $\operatorname{MSE}\left(\hat{\mu}_{\text {expz }}\right)-\operatorname{Min} \cdot \operatorname{MSE}\left(\hat{\mu}_{\text {expz }}\right)=T>0$, always if $0 \leq W<1$, and $\operatorname{MSE}\left(\hat{\mu}_{\exp Z}\right)=\operatorname{Min} \cdot \operatorname{MSE}\left(\hat{\mu}_{\exp z}\right)$ if $W=1 \quad($ as $0 \leq W \leq 1)$

(V) $\operatorname{MSE}\left(\hat{\mu}_{y z}\right)-\operatorname{Min} \cdot \operatorname{MSE}\left(\hat{\mu}_{\text {expz }}\right)=\lambda \bar{Y}^{2} C_{y}^{2} \rho_{y x}^{2}+T_{1 z}+T_{2 z}>0$, always.

(VI) $\operatorname{MSE}\left(\hat{\mu}_{R Z}\right)-\operatorname{Min} \cdot \operatorname{MSE}\left(\hat{\mu}_{\text {expz }}\right)=\lambda \bar{Y}^{2}\left(\rho_{y x} C_{y}-C_{x}\right)^{2}+T_{1 z}+T_{2 z}>0$, always.

(VII) $\operatorname{MSE}\left(\hat{\mu}_{\text {Regz }}\right)-\operatorname{Min} \cdot \operatorname{MSE}\left(\hat{\mu}_{\operatorname{expz}}\right)=T_{1 \mathrm{z}}+T_{2 Z}>0$, always.

(VIII) $\operatorname{MSE}\left(\hat{\mu}_{y Z}\right)-\operatorname{Min} \cdot \operatorname{MSE}\left(\hat{\mu}_{G R R Z}\right)=\frac{\bar{Y}^{2} C_{z}^{2} \lambda\left\{\left(1-\lambda C_{x}^{2}\right) \rho_{z x}^{2}+\lambda C_{z}^{2}\left(1-\rho_{z x}^{2}\right)\right\}}{\lambda C_{z}^{2}\left(1-\rho_{z x}^{2}\right)+\left(1-\lambda C_{x}^{2}\right)}+D>0$, provided that $\lambda C_{x}^{2}<1$ and $W<1$. 
(IX) $\operatorname{MSE}\left(\hat{\mu}_{R Z}\right)-\operatorname{Min} . \operatorname{MSE}\left(\hat{\mu}_{G R R Z}\right)=\bar{Y}^{2} C_{z}^{2} \lambda\left\{\left(\frac{C_{x}}{C_{z}}-\rho_{z x}\right)^{2}+\frac{\lambda C_{z}^{2}\left(1-\rho_{z x}^{2}\right)^{2}}{\lambda C_{z}^{2}\left(1-\rho_{z x}^{2}\right)+\left(1-\lambda C_{x}^{2}\right)}\right\}+D>0$,

provided that $\lambda C_{x}^{2}<1$ and $W<1$.

(X) $\operatorname{MSE}\left(\hat{\mu}_{R e g Z}\right)-\operatorname{Min} \cdot \operatorname{MSE}\left(\hat{\mu}_{G R R Z}\right)=\frac{\lambda^{2} \bar{Y}^{2} C_{Z}^{4}\left(1-\rho_{Z x}^{2}\right)^{2}}{\lambda C_{Z}^{2}\left(1-\rho_{z x}^{2}\right)+\left(1-\lambda C_{x}^{2}\right)}+D>0$, provided that $\lambda C_{x}^{2}<1$ and $W<1$.

(XI) $\operatorname{MSE}\left(\hat{\mu}_{G R R Z}\right)-\operatorname{Min} \cdot \operatorname{MSE}\left(\hat{\mu}_{G R R Z}\right)=D>0$, provided that $\lambda C_{x}^{2}<1$ and $W<1$.

where $D=\frac{\bar{Y}^{2}\left(1-\lambda C_{x}^{2}\right)^{2} \lambda C_{y}^{2}(1-W) S_{s}^{2}}{S_{y}^{2}\left\{\lambda\left(C_{y}^{2}+\frac{S_{s}^{2}}{\bar{Y}^{2}}\right)\left(1-\frac{\rho_{y x}^{2}}{1+\frac{S_{s}^{2}}{S_{y}^{2}}}\right)+\left(1-\lambda C_{x}^{2}\right)\right\}\left\{\lambda\left(C_{y}^{2}+W \frac{S_{s}^{2}}{\bar{Y}^{2}}\right)\left(1-\frac{\rho_{y x}^{2}}{1+W \frac{S_{s}^{2}}{S_{y}^{2}}}\right)+\left(1-\lambda C_{x}^{2}\right)\right\}}$.

(XII) $\operatorname{MSE}\left(\hat{\mu}_{y z}\right)-\operatorname{Min} \cdot \operatorname{MSE}\left(\hat{\mu}_{G R R Z}\right)=\frac{\bar{Y}^{2} C_{z}^{2} \lambda\left\{\left(1-\lambda C_{x}^{2}\right) \rho_{z x}^{2}+\lambda C_{z}^{2}\left(1-\rho_{z x}^{2}\right)\right\}}{\lambda C_{z}^{2}\left(1-\rho_{z x}^{2}\right)+\left(1-\lambda C_{x}^{2}\right)}>0$,

provided that $\lambda C_{x}^{2}<1$.

(XIII) $\operatorname{MSE}\left(\hat{\mu}_{R Z}\right)-\operatorname{Min} \cdot \operatorname{MSE}\left(\hat{\mu}_{G R R z}\right)=\bar{Y}^{2} C_{\mathrm{z}}^{2} \lambda\left\{\left(\frac{C_{x}}{C_{z}}-\rho_{\mathrm{z} x}\right)^{2}+\frac{\lambda C_{\mathrm{z}}^{2}\left(1-\rho_{\mathrm{z} x}^{2}\right)^{2}}{\lambda C_{z}^{2}\left(1-\rho_{z x}^{2}\right)+\left(1-\lambda C_{x}^{2}\right)}\right\}>0$, provided that $\lambda C_{x}^{2}<1$.

$(\mathrm{XIV}) \operatorname{MSE}\left(\hat{\mu}_{R e g z}\right)-\operatorname{Min} \cdot \operatorname{MSE}\left(\hat{\mu}_{G R R Z}\right)=\frac{\lambda^{2} \bar{Y}^{2} C_{z}^{4}\left(1-\rho_{z x}^{2}\right)^{2}}{\lambda C_{z}^{2}\left(1-\rho_{z x}^{2}\right)+\left(1-\lambda C_{x}^{2}\right)}>0$,

provided that $\lambda C_{x}^{2}<1$.

$(\mathrm{XV}) \operatorname{Min} \cdot \operatorname{MSE}\left(\hat{\mu}_{G R R Z}\right)-\operatorname{Min} \cdot \operatorname{MSE}\left(\hat{\mu}_{\exp Z}\right)>0$, provided that

$\frac{\lambda C_{x}^{2}\left\{\frac{\lambda}{16} C_{x}^{2}+\frac{M S E\left(\hat{\mu}_{\text {Regz }}\right)}{\bar{Y}^{2}}\right\}+4}{4\left(1+\frac{M S E\left(\hat{\mu}_{\text {Regz }}\right)}{\bar{Y}^{2}}\right)}+\frac{\frac{M S E\left(\widehat{\mu}_{R e g z}\right)}{\bar{Y}^{2}}\left(1-\lambda C_{x}^{2}\right)}{\frac{M S E\left(\widehat{\mu}_{R e g z}\right)}{\bar{Y}^{2}}+\left(1-\lambda C_{x}^{2}\right)}>1$.

From the above results, we note the following observations:

(A) Our proposed estimator $\hat{\mu}_{\text {expz }}$ is more efficient than the various existing estimators discussed in this article.

(B) The estimator $\hat{\mu}_{G R R z}$ is more efficient than estimators $\hat{\mu}_{y z}, \hat{\mu}_{R z}$ and $\hat{\mu}_{R e g z}$ under the condition $\lambda C_{x}^{2}<1$ and also more efficient than $\hat{\mu}_{y z}, \hat{\mu}_{R Z}, \hat{\mu}_{R e g z}$ and $\hat{\mu}_{G R R Z}$ under the conditions $W<1$ and $\lambda C_{x}^{2}<1$.

(C) It is interesting to note that the condition $\lambda C_{x}^{2}<1$ is very likely to hold true and also in the present paper, the condition $0 \leq W \leq 1$ is always true.

(D) The proposed estimator $\hat{\mu}_{\operatorname{expz}}$ in the Section 3 is more efficient than the other proposed estimator $\hat{\mu}_{G R R Z}$ if the condition (7) hold.

\section{Remarks 4.1:}


As we know that, bias has negligible impact on the accuracy of an estimator when the bias is less than one tenth of the standard deviation of estimator. We can be certain that the proportion Bias / St. Dev will not surpass 0.1 if the sample size is sufficiently large. So in the above comparison, we have considered only mean square errors of various estimators and not taking their biases [see pages 14-15 of Cochran (1977)].

\section{Numerical illustration}

We compare the efficiencies of various estimators numerically by using the some empirical populations. We obtain the percent relative efficiencies (PRE) of various estimators, with respect to $\hat{\mu}_{Y Z}$ by using the formula $\operatorname{PRE}\left(\hat{\mu}_{i}\right)=\frac{M S E\left(\hat{\mu}_{Y Z}\right)}{M S E\left(\widehat{\mu}_{i}\right)} \times 100, i=Y Z$, $Y Z, R Z, R Z, \operatorname{Reg} Z, \operatorname{Reg} Z, G R R Z, G R R Z, \exp Z, \exp Z$. The distribution of $S$ is taken to be normal with mean zero and standard deviation equal to $\alpha$ times the standard deviation of $X$ i.e. $S_{s}=\alpha \times S_{x}$ where $\alpha$ is scalar e.g. $\alpha=0.1,0.2$ and 0.3 .

Population I \{Source: Koyuncu et al. (2014)\}

$N=5336, \rho_{y x}=0.9632, \bar{X}=22.99, \bar{Y}=30.19, \quad S_{x}=172.09, S_{y}=138.65, \quad$ and $n=500$.

Table 5.1: Percent relative efficiencies of various estimators with respect to $\widehat{\mu}_{Y Z}$. with $\alpha=10 \%$

\begin{tabular}{|c|c|c|c|c|c|c|c|c|c|c|}
\hline 3 & 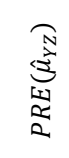 &  &  & 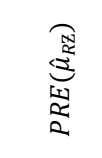 & 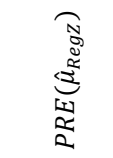 & 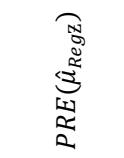 & 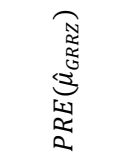 & 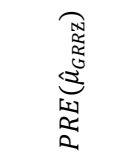 &  &  \\
\hline 0.1 & \multirow{10}{*}{100} & 101.38 & \multirow{10}{*}{190.82} & 195.92 & \multirow{10}{*}{1158.46} & 1376.14 & \multirow{10}{*}{1162.78} & 1380.46 & \multirow{10}{*}{1254.54} & 1504.16 \\
\hline 0.2 & & 101.23 & & 195.34 & & 1348 & & 1352.32 & & 1471.61 \\
\hline 0.3 & & 101.07 & & 194.76 & & 1320.98 & & 1325.3 & & 1440.44 \\
\hline 0.4 & & 100.92 & & 194.19 & & 1295.03 & & 1299.35 & & 1410.57 \\
\hline 0.5 & & 100.76 & & 193.62 & & 1270.08 & & 1274.4 & & 1381.91 \\
\hline 0.6 & & 100.61 & & 193.05 & & 1246.06 & & 1250.39 & & 1354.40 \\
\hline 0.7 & & 100.46 & & 192.49 & & 1222.95 & & 1227.27 & & 1327.97 \\
\hline 0.8 & & 100.30 & & 191.93 & & 1200.67 & & 1204.99 & & 1302.55 \\
\hline 0.9 & & 100.15 & & 191.37 & & 1179.19 & & 1183.51 & & 1278.09 \\
\hline 1 & & 100 & & 190.82 & & 1158.46 & & 1162.78 & & 1254.54 \\
\hline
\end{tabular}


Table 5.2: Percent relative efficiencies of various estimators with respect to $\widehat{\mu}_{Y Z}$ with $\alpha=\mathbf{2 0} \%$

\begin{tabular}{|c|c|c|c|c|c|c|c|c|c|c|}
\hline 3 & 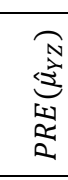 & 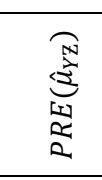 &  & 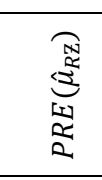 & 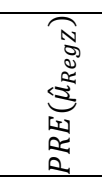 & 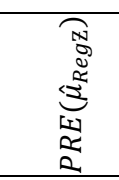 & 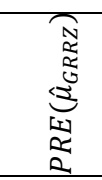 & 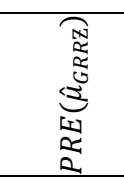 & 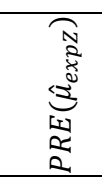 & 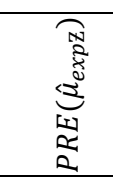 \\
\hline 0.1 & \multirow{10}{*}{100} & 105.51 & \multirow{10}{*}{183.56} & 203.03 & \multirow{10}{*}{793.04} & 1353.97 & \multirow{10}{*}{797.56} & 1358.49 & \multirow{10}{*}{845.18} & 1474.77 \\
\hline 0.2 & & 104.87 & & 200.66 & & 1255.32 & & 1259.83 & & 1361.84 \\
\hline 0.3 & & 104.24 & & 198.35 & & 1170.06 & & 1174.58 & & 1265.02 \\
\hline 0.4 & & 103.61 & & 196.1 & & 1095.65 & & 1100.17 & & 1181.08 \\
\hline 0.5 & & 102.99 & & 193.89 & & 1030.14 & & 1034.65 & & 1107.63 \\
\hline 0.6 & & 102.38 & & 191.73 & & 972.015 & & 976.533 & & 1042.8 \\
\hline 0.7 & & 101.77 & & 189.62 & & 920.103 & & 924.62 & & 985.171 \\
\hline 0.8 & & 101.17 & & 187.56 & & 873.454 & & 877.972 & & 933.6 \\
\hline 0.9 & & 100.58 & & 185.54 & & 831.308 & & 835.825 & & 887.179 \\
\hline 1 & & 100 & & 183.56 & & 793.041 & & 797.558 & & 845.175 \\
\hline
\end{tabular}

Table 5.3: Percent relative efficiencies of various estimators with respect to $\widehat{\boldsymbol{\mu}}_{Y Z}$ with $\alpha=\mathbf{3 0} \%$

\begin{tabular}{|c|c|c|c|c|c|c|c|c|c|c|}
\hline 3 & 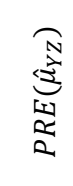 & 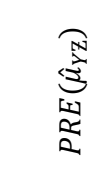 & 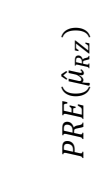 & 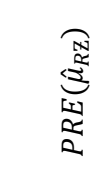 & 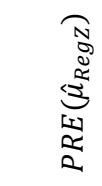 & 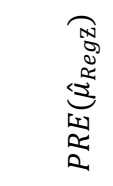 & 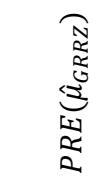 &  &  & 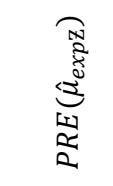 \\
\hline 0.1 & \multirow{10}{*}{100} & 112.31 & \multirow{10}{*}{173.74} & 214.6 & \multirow{10}{*}{539.92} & 1322.31 & \multirow{10}{*}{544.76} & 1327.15 & \multirow{10}{*}{570.14} & 1433.22 \\
\hline 0.2 & & 110.79 & & 209.13 & & 1138.93 & & 1143.77 & & 1226.12 \\
\hline 0.3 & & 109.32 & & 203.94 & & 1000.22 & & 1005.06 & & 1071.45 \\
\hline 0.4 & & 107.88 & & 199 & & 891.624 & & 896.469 & & 951.549 \\
\hline 0.5 & & 106.48 & & 194.29 & & 804.301 & & 809.147 & & 855.872 \\
\hline 0.6 & & 105.12 & & 189.8 & & 732.558 & & 737.403 & & 777.753 \\
\hline 0.7 & & 103.79 & & 185.51 & & 672.565 & & 677.41 & & 712.764 \\
\hline 0.8 & & 102.5 & & 181.42 & & 621.654 & & 626.499 & & 657.852 \\
\hline 0.9 & & 101.23 & & 177.49 & & 577.909 & & 582.754 & & 610.841 \\
\hline 1 & & 100 & & 173.74 & & 539.92 & & 544.76 & & 570.14 \\
\hline
\end{tabular}

Population II Source: Sousa et al. (2010)

$N=1000, \quad \rho_{y x}=0.8783, \quad \bar{X}=2, \quad \bar{Y}=2, \quad S_{x}=2.4495, \quad S_{y}=1.4142$ and $n=$ 50 . 
Table 5.4: Percent relative efficiencies of various estimators with respect to $\widehat{\mu}_{Y Z}$ with $\alpha=10 \%$

\begin{tabular}{|c|c|c|c|c|c|c|c|c|c|c|}
\hline 3 & $\begin{array}{l}\underset{N}{5} \\
\frac{5}{2} \\
\frac{1}{2}\end{array}$ & 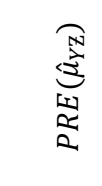 & $\begin{array}{l}\frac{\sqrt{N}}{2} \\
\frac{\sqrt{2}}{2} \\
\frac{1}{2} \\
\frac{1}{2}\end{array}$ & 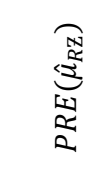 & 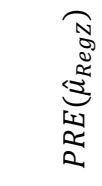 & 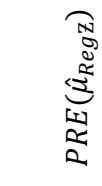 &  & 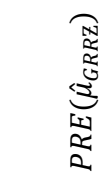 & 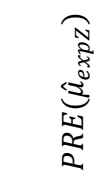 & 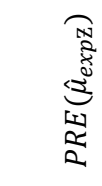 \\
\hline 0.1 & \multirow{10}{*}{100} & 102.69 & \multirow{10}{*}{104.3} & 107.23 & \multirow{10}{*}{398.31} & 444.75 & \multirow{10}{*}{399.32} & 445.76 & \multirow{10}{*}{404.26} & 451.55 \\
\hline 0.2 & & 102.39 & & 106.9 & & 439.07 & & 440.07 & & 445.76 \\
\hline 0.3 & & 102.08 & & 106.57 & & 433.52 & & 434.53 & & 440.11 \\
\hline 0.4 & & 101.78 & & 106.24 & & 428.12 & & 429.12 & & 434.6 \\
\hline 0.5 & & 101.48 & & 105.91 & & 422.84 & & 423.85 & & 429.23 \\
\hline 0.6 & & 101.18 & & 105.58 & & 417.7 & & 418.71 & & 424 \\
\hline 0.7 & & 100.88 & & 105.26 & & 412.68 & & 413.68 & & 418.88 \\
\hline 0.8 & & 100.59 & & 104.94 & & 407.78 & & 408.78 & & 413.89 \\
\hline 0.9 & & 100.29 & & 104.62 & & 402.99 & & 404 & & 409.02 \\
\hline 1 & & 100 & & 104.3 & & 398.31 & & 399.32 & & 404.26 \\
\hline
\end{tabular}

Table 5.5: Percent relative efficiencies of various estimators with respect to $\widehat{\mu}_{Y Z}$ with $\alpha=20 \%$

\begin{tabular}{|c|c|c|c|c|c|c|c|c|c|c|}
\hline 3 & 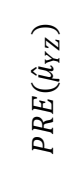 & 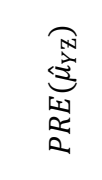 & 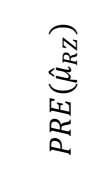 & 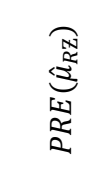 & 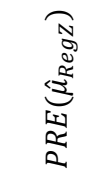 & 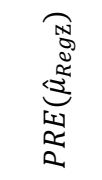 & 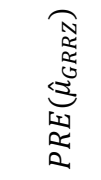 & 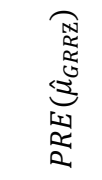 & 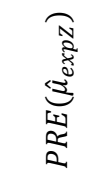 & 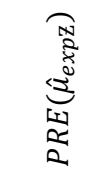 \\
\hline 0.1 & \multirow{10}{*}{100} & 110.67 & \multirow{10}{*}{103.94} & 115.52 & \multirow{10}{*}{321.29} & 465.52 & \multirow{10}{*}{322.39} & 466.62 & \multirow{10}{*}{325.93} & 472.58 \\
\hline 0.2 & & 109.38 & & 114.11 & & 443.41 & & 444.5 & & 450.06 \\
\hline 0.3 & & 108.11 & & 112.73 & & 423.3 & & 424.39 & & 429.59 \\
\hline 0.4 & & 106.87 & & 111.39 & & 404.93 & & 406.03 & & 410.91 \\
\hline 0.5 & & 105.66 & & 110.07 & & 388.09 & & 389.19 & & 393.79 \\
\hline 0.6 & & 104.48 & & 108.79 & & 372.6 & & 373.7 & & 378.04 \\
\hline 0.7 & & 103.32 & & 107.54 & & 358.3 & & 359.39 & & 363.5 \\
\hline 0.8 & & 102.19 & & 106.31 & & 345.05 & & 346.15 & & 350.05 \\
\hline 0.9 & & 101.08 & & 105.11 & & 332.75 & & 333.84 & & 337.56 \\
\hline 1 & & 100 & & 103.94 & & 321.29 & & 322.39 & & 325.93 \\
\hline
\end{tabular}


Table 5.6: Percent relative efficiencies of various estimators with respect to $\widehat{\mu}_{Y Z}$ with $\alpha=30 \%$

\begin{tabular}{|c|c|c|c|c|c|c|c|c|c|c|}
\hline 3 & 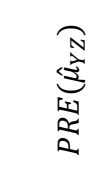 &  & 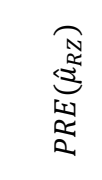 & $\begin{array}{l}\frac{2}{2} \\
\frac{2}{2} \\
\frac{1}{2} \\
\frac{1}{2}\end{array}$ & 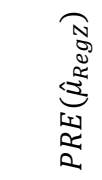 & 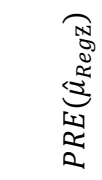 & 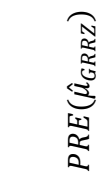 & 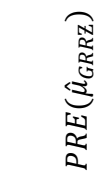 &  & 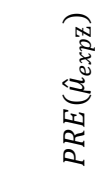 \\
\hline 0.1 & \multirow{10}{*}{100} & 123.66 & \multirow{10}{*}{103.46} & 129 & \multirow{10}{*}{254.72} & 496.89 & \multirow{10}{*}{255.96} & 498.13 & \multirow{10}{*}{258.46} & 504.33 \\
\hline 0.2 & & 120.49 & & 125.55 & & 449.42 & & 450.66 & & 456.03 \\
\hline 0.3 & & 117.48 & & 122.29 & & 410.22 & & 411.46 & & 416.19 \\
\hline 0.4 & & 114.62 & & 119.19 & & 377.31 & & 378.56 & & 382.77 \\
\hline 0.5 & & 111.89 & & 116.25 & & 349.29 & & 350.54 & & 354.33 \\
\hline 0.6 & & 109.29 & & 113.44 & & 325.15 & & 326.39 & & 329.83 \\
\hline 0.7 & & 106.81 & & 110.77 & & 304.12 & & 305.37 & & 308.52 \\
\hline 0.8 & & 104.44 & & 108.22 & & 285.65 & & 286.9 & & 289.8 \\
\hline 0.9 & & 102.17 & & 105.79 & & 269.3 & & 270.54 & & 273.23 \\
\hline 1 & & 100 & & 103.46 & & 254.72 & & 255.96 & & 258.46 \\
\hline
\end{tabular}

From the Table 5.1 to Table 5.6, we observe the following facts:

(i) The percent relative efficiencies of the all estimators with optional RRT decrease as the value of $W$ increases.

(ii) The proposed estimator $\hat{\mu}_{\text {expz }}$ is always more efficient than the various existing estimators considered in this paper.

(iii) It is important to note that various estimators with optional RRT model are always more efficient than the corresponding estimator with traditional RRT model.

(iv) The estimators with optional RRT are equally efficient to their corresponding estimators with traditional RRT model only when $W=1$. (see Remark: 2.1 )

6. Conclusion: By applying optional RRT model in the estimator of Koyuncu et al (2014), we not only improve the efficiency of estimator suggested by Koyuncu et al (2014) but also obtain an estimator which is more efficient than Gupta et al (2014)'s estimators based on optional RRT model.

Acknowledgements: Authors are thankful to the editor and the two unknown learned referees for their useful and encouraging comments and suggestions, which led to the present improved version of the paper.

\section{References}

1. Bahl, S. and Tuteja, R.K. (1991). Ratio and product type exponential estimators. Information and Optimization Sciences, 12(1):159-163.

2. Cochran, W.G. (1977). Sampling Techniques. Third Edition, New Delhi, India: Wiley Eastern Limited

3. Grover, L.K. and Kaur, P. (2011). An improved estimator of the finite population mean in simple random sampling. Model Assisted Statistics and Applications, 6(1): 47-55. 
4. Gupta, S., Kalucha, G., Shabbir, J. and Dass, B.K. (2014). Estimation of finite population mean using Optional RRT Models in the presence of non sensitive auxiliary information. American journal of Mathematical and Management Sciences, 33:147-159.

5. Gupta, S., Mehta, S., Shabbir, J. and Dass, B.K. (2013). Generalized scrambling in quantitative optional randomized response models. Communications in Statistics- Theory and Methods. 42(20):1-9.

6. Gupta, S., Shabbir, J. and Sehra, S. (2010). Mean and sensitivity estimation in optional randomized response models. Journal of Statistical and Planning Inference. 140(10):2870-2874.

7. Gupta, S., Shabbir, J., Sousa, R. and Corte-Real, P. (2012). Estimation of the mean of a sensitive variable in the presence of auxiliary information. Communication in Statistics- Theory and Methods. 41:2394-2404.

8. Huang, K.C. (2010). Unbiased estimators of mean, variance and sensitivity level for quantitative characterstics in finite population sampling. Metrika. 71:341-352.

9. Kalucha, G., Gupta, S. and Dass, B.K. (2015). Ratio estimation of finite population mean using optional randomized response models. Journal of Statistical Theory and Practice. 9:633-645.

10. Koyuncu, N., Gupta, S. and Sousa, R. (2014). Exponential-type estimators of the mean of a sensitive variable in the presence of non sensitive auxiliary information. Communications in statistics- Simulation and Computation, 43: 1583-1594.

11. Singh, H.P. and Solanki, R.S. (2012). Improved estimation of population mean in simple random sampling using information on auxiliary attribute. Applied Mathematics and Computation. 218:7798-7812.

12. Singh, H.P. and Tarray, T.A. (2014). A stratified Mangat and Singh's optional randomized response model using proportional and optimal allocation. Statistica, anno. 74(1):65-83.

13. Sousa, R., Shabbir, J., Corte-Real, P., and Gupta, S., (2010). Ratio estimation of the mean of a sensitive variable in the presence of auxiliary information. Journal of Statistical Theory and Practice. 4(3):495-507.

14. Tarray, T.A., Singh, H.P. and Zaizai, Y. (2015). A dexterous optional randomized response model. Sociological Methods and Research. 46(3): 565-585.

15. Tarray, T.A. and Singh, H.P. (2017). An improved estimation procedure of the mean of a sensitive variable using auxiliary information. Bio-Statistics and Biometrics. 3(2):1-8 